\title{
The Effects of the Contraction Degree of Hip Joint Adductor on Abdominal Muscle Activity during Bilateral Lower Extremity Raising
}

\author{
Min-Kyu, Kim¹, Yong-Ho Cho², Ji-Won Park', Jin-Ho Choi², Yu-Min Ko ${ }^{4}$ \\ 'Department of Physical Therapy, General Graduate School, Catholic University of Daegu, Daegu; ${ }^{2}$ Department of Physical Therapy, College of \\ Biomedical Science, Daegu Haany University, Daegu; ${ }^{3}$ Department of Physical Therapy, College of Biomedi, Catholic University of Daegu, Daegu; \\ ${ }^{4}$ Department of Physical Therapy, Gangneung Yeongdong College, Gangneung, Korea
}

Purpose: This study was to investigate the differences abdominal muscles activities of according to hip adductor contraction levels $20 \%$ (mild), 50\% (moderate), and 70\% (strong) of MVIC on during bilateral lower extremity raising exercise on supine.

Methods: The subjects of the study were a total of 39 persons including 23 healthy males and 16 females, who performed bilateral lower extremity raising exercise in 20\%,50\%, 70\% MVIC hip contraction. Muscle activities were measured by using S-EMG in RA (rectus abdominis), 10 (internal oblique), and EO (external oblique).

Results: Muscle activity of the internal oblique abdominal muscle and external oblique abdominal muscle, their activities were also greatest with the adductor contraction size at 70\% and there was statistically significant difference when compared with the adductor contraction size at $20 \%$ and $50 \%(\mathrm{p}<0.05)$. As for the rectus abdominis muscle according to the size of contraction of the adductor was greatest at 70\%, without statistically significant difference ( $p>0.05)$.

Conclusion: bilateral lower extremity raising with strong hip joint adductor contraction was effective exercise to strengthen abdominal muscles. If subjects could not perform strong hip adductor contraction, moderate contraction is effective abdominal muscle contraction exercise. The contraction size of the adductor is small, weak contraction may trigger middle level contraction and therefore appropriate application of the exercise program of bilateral leg raising may result in great effect as a lumbar stabilization exercise.

Keywords: Abdominal muscles, Adductor, EMG

\section{INTRODUCTION}

A major cause of low back pain is abnormality and inappropriate harmony of the muscles that support the body centered on the trunk and the pelvis. ${ }^{1,2}$ Low back pain is a very common disease over $80 \%$ of the population undergoes during a lifetime. ${ }^{3}$ In economic terms, the occurrence frequency of the disease has greatly increased, accounting for a large portion of medical costs and the disease has become a social problem with growing seriousness. The therapeutic methods to treat low back pain are physical therapies. They include manual therapy as well as thermal therapy, light therapy, and electrical therapy. Tai-chi is good method for decreasing low

Received Jun 2, 2016 Revised Jun 21, 2016

Accepted Jun 29, 2016

Corresponding author Ji-Won Park

E-mail mylovept@hanmail.net back pain. ${ }^{4}$

In exercise therapies including manual therapy, lumbar stabilization acts as a very important element in managing low back pain and in such stabilization of the lumbar region, muscle strength and endurance of the trunk and the pelvic muscles are very important. ${ }^{5}$ When muscle strength and endurance of the lumbar region are sufficient, diverse motions and movements are possible during daily life and in particular, the rectus abdominis muscle, external oblique abdominal muscle, and internal oblique abdominal muscle among the abdominal muscles act symmetrically and sequentially, triggering natural movement of the body.

The rectus abdominis muscle and external oblique abdominal

Copylight (C2016 The Korea Society of Physical Therapy

This is an Open Access article distribute under the terms of the Creative Commons Attribution Non-commercial License (Http:// creativecommons.org/license/by-nc/4.o.) which permits unrestricted non-commercial use, distribution, and reproduction in any medium, provided the original work is properly cited. 
muscle are superficial muscles and trigger great force and the internal oblique abdominal muscle is a deep muscle contributing to trunk stability together with the superficial muscles. ${ }^{7}$ The abdominal muscles when contracting simultaneously with the pelvic floor muscles act essentially for increased pressure in the abdomen, thereby decreasing pressure given to the waist and increasing stability. ${ }^{8}$ In addition, the abdominal muscles trigger movement such as flexion and rotation through contraction and contribute to appropriate distribution of excessive load given to the trunk to the lumbar bones and hip joints and act importantly in movements of the extremities and respiration. ${ }^{9}$ Together with the important acting of the abdominal muscles in stability, when the weight of the lower extremities is supported and the adductor and the abductor accounting for about a fourth of the thigh muscles make balance of contraction appropriately, stability is maintained and the pelvis is maintained symmetrically, forming balance of the body. ${ }^{10}$ As examined above, the strengthening of the abdominal muscle is very important for trunk stabilization and diverse methods are used for strengthening the abdominal muscles.

A method to strengthen the abdominal muscles without any tool includes bilateral lower extremity raising. As it was shown above that the adductor acts as an important element to support lower limb weight, contraction of the hip joint adductor during bilateral lower extremity raising is considered to give external load to the abdominal muscles affecting the size of contraction. However, research on the size of the contraction of the hip joint adductor and abdominal muscle activity is very lacking and therefore such research is considered necessary. Accordingly, this study was conducted in order to provide a more efficient method during abdominal muscle strengthening training in rehabilitation exercise programs.

\section{METHODS}

\section{Subjects}

The subjects of the current study are a total of 39 persons including 23 healthy males and 16 females. Before the experiment, the subjects were briefed on the purpose and methods of the study, and the experiment proceeded with those who voluntarily agreed to participate. The subjects were asked to fill out and submit the statement of consent in accordance with the ethical principles for medical research involving human subjects of the 'Declaration of Helsinki' be- fore the experiment. In terms of the protocol, the current study obtained approval from the Ethics Committee of Catholic University of Daegu to conduct the experiment. The average age of the subjects was 25.4; the average height $170.2 \mathrm{~cm}$; the average weight $64.6 \mathrm{~kg}$; and the average BMI (Korean Dietetic Association, 2008) was 22.14 $\mathrm{kg} / \mathrm{m}^{2}$.

\section{Experimental methods \\ 1) Measurement}

(1) Goniometer

The subjects basically raised the legs with knee extension and hip joint flexion angle at 30 degrees using a joint goniometer. In order to prevent increase in lumbar lordosis, the drawing-in contraction method of the abdomen was employed and activity of abdominal muscles was measured by differently contracting the hip joint adductor with the legs raised.

\section{(2) Stabilizer pressure bio-feedback}

The adductor contraction was measured at 20\% (weak), 50\% (moderate), and 70\% (strong) of the maximal pressure power. ${ }^{11}$ The size of contraction of the hip joint adductor was measured using a stabilizer pressure bio-feedback (CHATANOOGA Group Inc., USA).

\section{(3) Electromyography}

The Muscle activity was measured electromyography and Ag-Ag/ $\mathrm{Cl}$ electrode was used. Muscle activity was measured with WEMG8 (LXM5308, LAXTHA, Korea), a wireless electromyography equipment. The muscles activity was measured included the rectus abdominis muscle, external oblique abdominal muscle, and internal oblique abdominal muscle. The muscles of the dominant side were measured. The electrodes were attached to the belly in the middle between the naval and pubic bone for the rectus abdominis muscle, $15 \mathrm{~cm}$ outside from the navel height for the external oblique abdominal muscle, and the region in the middle between ASIS and pubic symphysis for the internal oblique abdominal muscle. ${ }^{12}$ In order to obtain the maximal isometric contraction value, \%MVIC based on manual muscle test was used. The motion of raising the legs for seven seconds was measured. The data value for the five seconds excluding the first second and the last second was used. In order to reduce the variable of fatigue resulting from exercise, the subjects took a rest for one minute between each motion. Each motion 
was performed three times and the average value was used for analysis. The motion order of the subjects was randomized in order to reduce variable according to measurement order. In order to reduce measurement noise, a notch filter at $60 \mathrm{~Hz}$ was used and data was processed using 20 to $500 \mathrm{~Hz}$ bandpass filtering.

\section{2) Statistical analysis}

The measured data was statistically processed using SPSS 19.0; in order compare measured values, repeated measures analysis of variance was conducted. Comparison of muscle activity was also executed using contrast test. Statistical significance level was $\alpha=0.05$.

\section{RESULTS}

Muscle activity of the internal oblique abdominal muscle and external oblique abdominal muscle, their activities were also greatest with the adductor contraction size at $70 \%$ and there was statistically significant difference when compared with the adductor contraction size at $20 \%$ and $50 \%(\mathrm{p}<0.05)$. As for the rectus abdominis muscle according to the size of contraction of the adductor was greatest at $70 \%$, without statistically significant difference $(\mathrm{p}>0.05)$ (Table 1).

\section{DISCUSSION}

The trunk muscle consists of large muscles on the surface and small muscles in the deep areas. The large muscles generate force and play the role of making large movements, delivering force from the upper and lower extremities and contribute to overall stability of the body. ${ }^{13}$ The small muscles do not greatly affect large movements of the body but play a major role in aiding joint stability together with an assistant role for movements. The internal oblique abdominal muscle is a representative small muscle in the trunk deep area, increases abdominal pressure together with the transversus abdomi-

Table 1. Differences of muscle activities according to contraction levels

\begin{tabular}{lccc}
\hline Muscle & $20 \%$ & $50 \%$ & $70 \%$ \\
\hline RA & $30.7 \pm 19.0$ & $30.1 \pm 21.6$ & $33.3 \pm 21.7$ \\
IO & $22.9 \pm 9.9^{+}$ & $23.9 \pm 9.5^{+}$ & $27.2 \pm 11.9$ \\
EO & $16.8 \pm 8.4^{+}$ & $16.9 \pm 8.2^{+}$ & $18.7 \pm 8.8$ \\
\hline
\end{tabular}

RA: Rectus abdominis, IO: Internal oblique, EO: External oblique. ${ }^{+}$significant difference between $20 \%$ and $70 \%$; ${ }^{*}$ significant difference between $50 \%$ and $70 \%$. nis muscle, and is conducive to rotating the trunk in the same direction through contraction. ${ }^{14}$ The body can make movement and heighten stability through overall harmony and coordination of such trunk muscles. ${ }^{15}$ However, when the muscles constituting the trunk such as the abdominal muscles become weak, anterior tilting occurs resulting from pelvic abnormality and lumbar lordosis increases, which may become the cause of low back pain.

In addition, when muscle imbalance leads to difference in the length of bilateral muscles, a serious problem may be triggered through imbalance of the pelvis and the spine. ${ }^{16}$ If the trunk area is stabilized through appropriate muscle strength and endurance of the trunk muscles, low back pain may be prevented and treated. In the past, in relation to low back pain, a main focus was on restriction to movement due to non-contractile tissues, which are ligaments and connective tissues, but recently integral action of the muscles with positive action and nerves with dominance is placed importance on all the more and for such reason exercises for stabilizing the trunk are conducted much. ${ }^{17}$ For pelvic stabilization regarding contraction of the hip joint muscles, contraction and adjustment of the abdominal muscles is necessary and efficient delivery to the lower limbs and the hip joints requires pelvic stability. Such stability may occur through the strengthening of the trunk muscles.

General trunk strengthening exercise includes sit-up, lumbar extensor strengthening exercise, and bilateral lower extremity raising. Among them, the first two exercises focus on the rectus abdominis muscle and erector spinae muscle, failing to play a great role in strengthening internal oblique abdominal muscle and external oblique abdominal muscle that are greatly conductive to stability. ${ }^{18}$ On the contrary, bilateral lower extremity raising in a supine position results in leg weight as external resistance, strengthening trunk muscles as an effective exercise for stabilization. ${ }^{19}$ As examined through previous studies, the abdominal muscles play a very important role in physical stability.

Accordingly, this study focused on the abdominal muscles. For an effective exercise to strengthen the abdominal muscles, abdominal muscle activity according to the degree of contraction of the hip joint adductor, which may be a great variable in abdominal muscle activity, was measured to examine the effects of hip joint adductor contraction on the strengthening of abdominal muscles. All the rectus abdominis muscle, internal oblique abdominal muscle, and 
external oblique abdominal muscle among the abdominal muscles had greatest activity at 70\% among the three exercise intensities.

In addition, in the case of internal oblique abdominal muscle and external oblique abdominal muscle, there was statistically significant difference between at 70\% contraction and at the other intensities. However, there was no statistically significant difference at $20 \%$ and $50 \%$. Further, in the case of the rectus abdominis muscle, there was greatest activity in 70\% but there was no statistical significance with the other contraction degrees. This is associated with the hip joint adductor's lower extremity movement and the function to support and fix the trunk. ${ }^{20}$

Previous studies showed the correlation between muscle activities of deep trunk muscles and the stabilities of back and pelvis. Especially, the back stability was increased as activity ratio of deep trunk muscles increased. ${ }^{21,22}$ Quadratus lumborum and psoasmajor connect hip adductors and deep trunk muscles, that means hip adductors affect the functions of deep trunk muscles and abdominal pressure. ${ }^{23}$ Because hip adductors contraction initiates the activations of pelvic and abdominal muscles, the abdominal pressure is increased. ${ }^{24,25}$

This study result suggests that during bilateral lower extremity raising exercise, strongly contracted hip joint adductor increases activity of the abdominal muscles conductive to stabilization of the lumbar region. However, it is considered that when the contraction size of the adductor is small, weak contraction may trigger middle level contraction and therefore appropriate application of the exercise program of bilateral leg raising may result in great effect as a lumbar stabilization exercise.

\section{REFERENCES}

1. Nelson-Wong E, Gregory DE, Winter DA et al. Gluteus medius muscle activation patterns as a predictor of low back pain during standing. Clin Biomech. 2008;23(5):545-53.

2. Houglum PA, Bertoti DB. Brunnstrom's clinical kinesiology. 6th ed, Philadelphia, FA Davis, 2011.

3. O'Sullivan PB. Lumbar segmental 'instability': clinical presentation and specific stabilizing exercise management. Man Ther. 2000;5(1):2-12.

4. Cho YH. Effects of tai chi on pain and muscle activity in young males with acute low back pain. J Phys Ther Sci. 2014;26(4):679-81.

5. Handa N, Yamamoto H, Tani T et al. The effect of trunk muscle exercises in patients over 40 years of age with chronic low back pain. J Orthop Sci. 2000;5(3):210-6.
6. Stevens VK, Coorevits PL, Bouche KG et al. The influence of specific training on trunk muscle recruitment patterns in healthy subjects during stabilization exercises. Man Ther. 2007;12(3): 271-9.

7. Lee HO. Activation of trunk muscles during stabilization exercises in four-point kneeling. J Kor Phys Ther. 2010;22(5):33-8.

8. Kim BK, Lee MH, Kim GC. Comparison of abdominal muscle activity during exercises using a sling. J Kor Phys Ther. 2012;25(3):149-54.

9. Neumann P, Gill V. Pelvic floor and abdominal muscle interaction: EMG activity and intra-abdominal pressure. Int Urogynecol J. 2002;13(2):12532.

10. Kapandji IA. The physiology of the joints volume 2. 6th ed, New York, Churchill Livingstone. 2005.

11. Wattanaprakornkul D, Cathers I, Halaki M et al. The rotator cuff muscle have a direction specific recruitment pattern during shoulder flexion and extension exercises. J Sci Med Sport. 2011;14(5):376-82.

12. Stevens VK, Coorevits PL, Bouche KG et al. The influence of specific training on trunk muscle recruitment patterns in healthy subjects during stabilization exercises. Man Ther. 2007;12(3):271-9.

13. Hodges PW, Moseley GL. Pain and motor control of the lumbopelvic region: effect and possible mechanisms. J Electromyogr Kinesiol. 2003; 13(4):361-70.

14. Houglum PA, Bertoti DB.Brunnstrom's clinical kinesiology.6th ed. Philadelphia. FA Davis. 2012.

15. Chang JS, Lee HY, Lee MY.The study of lumbar erector spinea and rectus abdominis activations according to the different gait velocities in young healthy adults. J Kor Phys Ther. 2012;24(3):186-90

16. Kendall FP, Mc Creary EK, Provance PG. Muscles; testing and function with posture and pain. 5th ed, Lippincott Williams\& Wilkins, 2005.

17. Murphy DR. Conservative management of cervical spine syndrome. USA, McGraw-Hill Companies Inc. 2000.

18. Vera-Garcia FJ, Grenier SG, McGill SM. Abdominal muscle response during curl-up on both stable and labile surfaces. Phys Ther. 2000; 80(6):564-9.

19. Richardson C, Jull G, Toppenberg R et al. Techniques for active lumbar stabilization for spinal protection: a pilot study. Aust J Physiother. 1992; 38(2):105-12.

20. Kibler WB, Chandler TJ, Livingston BP et al. Shoulder range of motion in elite tennis players effect of age and years of tournament play. Am J Sports Med. 1996;24(3):279-85.

21. Hodges PW, Richardson CA. Inefficient muscular stabilization of the lumbar spine associated with low back pain. A motor control evaluation of transversus abdominis. Spine(Phila Pa 1976). 1996;21(22):2640-50.

22. Arokoski JP, Valta T, Kankaanpa AM et al. Activation of lumbar paraspinal and abdominal muscles during therapeutic exercises in chronic low back pain patients. Arch Phys Med Rehabil. 2004;85(5):823-32.

23. Thomas WM. Anatomical Trains: Myofascial Meridians for Manual and Movement Therapists. 2nd ed. London. Churchill Livingstone. 2009.

24. Homborg B, Moritz U, Hamberg J et al. Intra-abdominal pressure and trunk muscle activity during lifting-effect of abdominal muscle training in healthy subjects. Scand J Rehabil Med. 1983;15(4):183-96.

25. Cholewicki J, Juluru K, McGill SM. Intra-abdominal pressure mechanism for stabilizing the lumbarspine. J Biomech. 1999;32(1):13-7. 